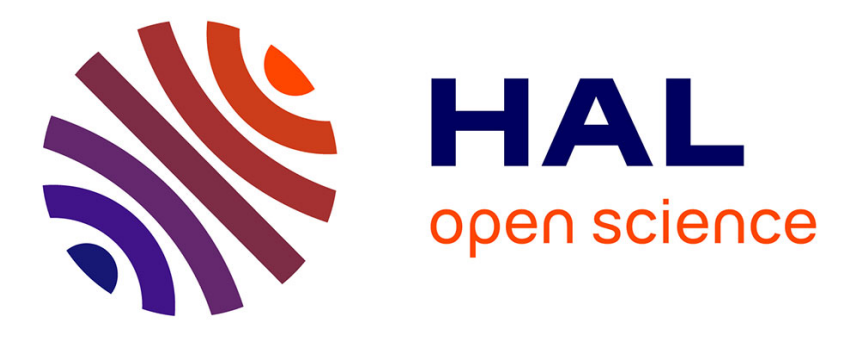

\title{
Nonlinear optical properties of interconnected gold nanoparticles on silicon
}

Antoine Lesuffleur, Philippe Gogol, Pierre Beauvillain, B. Guizal, Daniel van Labeke, Patrick Georges

\section{- To cite this version:}

Antoine Lesuffleur, Philippe Gogol, Pierre Beauvillain, B. Guizal, Daniel van Labeke, et al.. Nonlinear optical properties of interconnected gold nanoparticles on silicon. Journal of Applied Physics, 2008, 104, pp.124310. 10.1063/1.3050309 . hal-00691687

\section{HAL Id: hal-00691687 \\ https://hal-iogs.archives-ouvertes.fr/hal-00691687}

Submitted on 26 Apr 2012

HAL is a multi-disciplinary open access archive for the deposit and dissemination of scientific research documents, whether they are published or not. The documents may come from teaching and research institutions in France or abroad, or from public or private research centers.
L'archive ouverte pluridisciplinaire HAL, est destinée au dépôt et à la diffusion de documents scientifiques de niveau recherche, publiés ou non, émanant des établissements d'enseignement et de recherche français ou étrangers, des laboratoires publics ou privés. 


\title{
Nonlinear optical properties of interconnected gold nanoparticles on silicon
}

\author{
Antoine Lesuffleur, ${ }^{1, a)}$ Philippe Gogol, ${ }^{1}$ Pierre Beauvillain, ${ }^{1}$ B. Guizal, ${ }^{2}$ D. Van Labeke, ${ }^{2}$ \\ and P. Georges ${ }^{3}$ \\ ${ }^{1}$ Institut d'Electronique Fondamentale, UMR CNRS 8622, Université Paris-Sud Orsay, 91405 Orsay Cedex, \\ France \\ ${ }^{2}$ Insitut FEMTO-ST, UMR CNRS 6174, Université de Franche-Comte, Route de Gray, 25030 Besancon \\ Cedex, France \\ ${ }^{3}$ Institut d'Optique, UMR CNRS 8501, Campus Polytechnique, RD128, 91127 Palaiseau, France
}

(Received 15 September 2008; accepted 5 November 2008; published online 19 December 2008)

\begin{abstract}
We report second harmonic generation (SHG) measurements in reflectivity from chains of gold nanoparticles interconnected with metallic bridges. We measured more than 30 times a SHG enhancement when a surface plasmon resonance was excited in the chains of nanoparticles, which was influenced by coupling due to the electrical connectivity of the bridges. This enhancement was confirmed by rigorous coupled wave method calculations and came from high localization of the electric field at the bridge. The introduction of $10 \%$ random defects into the chains of nanoparticles dropped the SHG by a factor of 2 and was shown to be very sensitive to the fundamental wavelength. (C) 2008 American Institute of Physics. [DOI: 10.1063/1.3050309]
\end{abstract}

\section{INTRODUCTION}

Plasmonics is a rapidly emerging field in nanoscience as a subdiscipline of nanophotonics. One of its main features is to spatially confine high electric field at the nanoscale. Surface plasmons (SPs) are collective oscillations of free electrons in metals such as gold and silver. These oscillations can behave as waves at metal/dielectric interfaces and can be localized in metallic nanostructures such as nanoparticles.

Arrays of ordered gold nanoparticles have been investigated intensively during the past years. Coupling between the nanoparticles due to SP excitations has been studied theoretically and experimentally mostly by linear far-field spectroscopy $^{1-4}$ and by near-field microscopy. ${ }^{5,6}$ In those studies, it has been shown that a strong coupling between the particles can promote certain resonance orders compared to noncoupled particles. Significant spectral shifts were observed on linear far-field spectra depending on the spacing between the nanoparticles. Propagation of the SP excitation within nanoparticle chains due to near-field coupling has been demonstrated for nanoparticles spaced by a gap of a few tens of nanometers. ${ }^{7}$ In the simplest case, a single subwavelength nanoparticle, the SP resonance consists of a dipolar polarization of the particle. ${ }^{8}$ In regular arrays the nearfield coupling between the nanoparticles induces a redshift of the dipolar resonance and the appearance of higher order resonances (quadrupolar, etc.). ${ }^{2}$ Pairs of interconnected nanoparticles present stronger quadrupolar resonances and a larger redshift of the dipole resonance induced by the metallic connection between the particles compared to unconnected nanoparticles. ${ }^{3,4}$ All these studies have demonstrated that the SP excited in a particle is strongly dependent on the surrounding environment such as neighboring particles or the substrate.

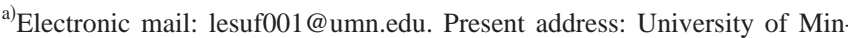
nesota, ECE Department, 200 Union Street SE, MN 55455, USA.
}

For exploring the enhancement in the electric field at the surface of metallic nanoparticles, second harmonic generation (SHG) is a useful technique since its efficiency is proportional to the square of the local field intensity. Although SHG is not possible in centrosymmetric media such as bulk gold, it may be observed at the surface where the symmetry is broken. It has been shown experimentally and theoretically that SHG can be highly enhanced by the SPs on rough metallic surface, ${ }^{9,10}$ single or randomly ordered metallic nanoparticles, ${ }^{10-12}$ and arrays of noncoupled metallic nanoparticles. ${ }^{13}$ Also, nanoapertures in a metallic film have been studied by SHG and a strong enhancement factor has been measured due to the high confinement of the electric field at the nanoscale. ${ }^{14,15}$ However, SHG from interconnected chains of nanoparticles has not been investigated yet.

In this work, we present SHG measurements from arrays of gold nanoparticle chains on a silicon substrate. The nanoparticles were interconnected by gold nanobridges in one direction. The chains were spaced by a $200 \mathrm{~nm}$ gap separation to avoid near-field coupling in the orthogonal direction. An enhancement was measured when SP resonances were excited at the SHG wavelength. Furthermore, a similar array with $10 \%$ of defects (missing nanoparticles) was fabricated, and a decrease in SHG was observed showing the significant role of the interaction between the resonant nanoparticles.

\section{EXPERIMENTAL TECHNIQUES}

Figure 1(a) shows a scanning electron microscope (SEM) image of an array of interconnected gold nanoparticle chains on a silicon substrate. The nanoparticles were fabricated by e-beam lithography combined with ion beam etching on a $50 \mathrm{~nm}$ thick gold layer deposited directly on the silicon substrate. Nanoparticle dimensions are 140 $\times 165 \mathrm{~nm}^{2}$ with a height of $50 \mathrm{~nm}$. The separation between neighboring chains is $200 \mathrm{~nm}$ and the gaps forming the 

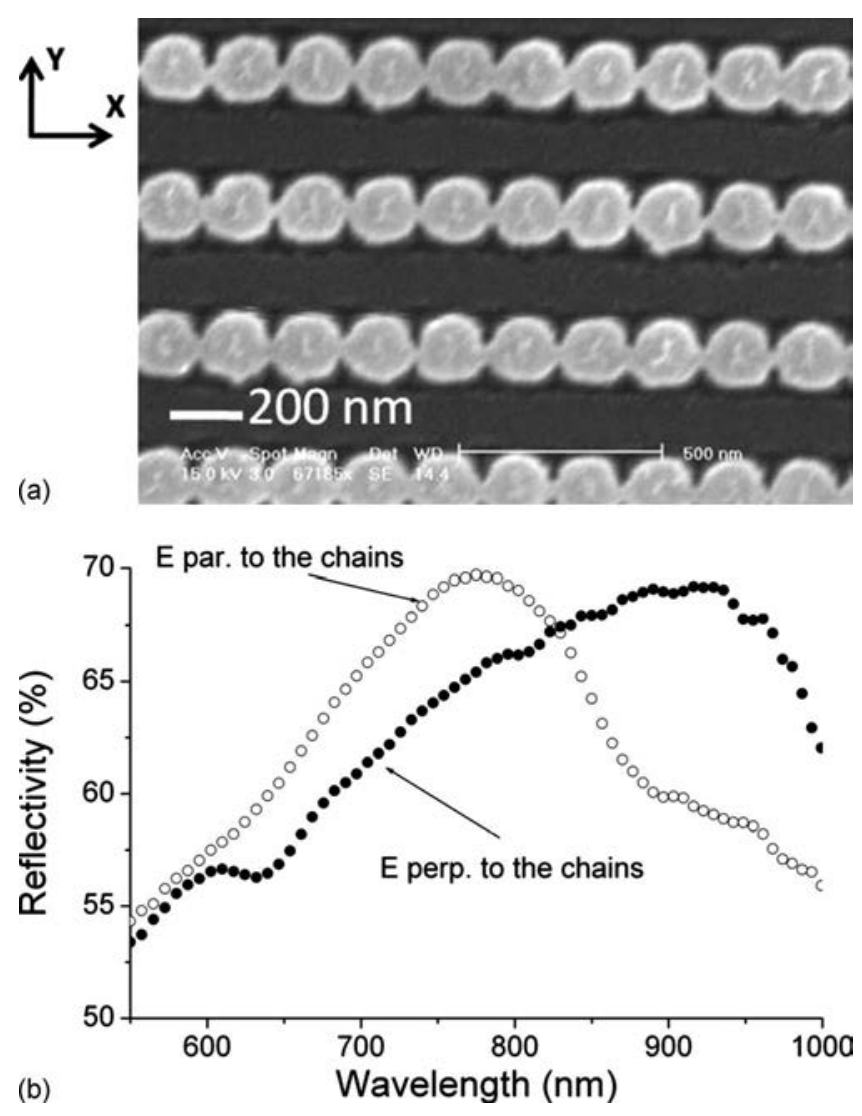

FIG. 1. (a) SEM image of the chains of gold nanoparticles. (b) Spectra in reflectivity of the chains of gold nanoparticles for two orientations of the incidence plane in TE polarization.

chains of nanoparticles are made with a 10-nm-long thin gold bridge. The size of the entire array of nanoparticle chains is $35 \times 60 \mu \mathrm{m}^{2}$. Two arrays (with and without defects) were made on the Si substrate using the same process.

Linear optic measurements were performed using a homemade reflectometer, a tungsten halogen source, and an ocean optics USB2000 spectrometer with a $45^{\circ}$ incidence angle. Spectral measurements are reported using a direct experimental comparison of the reflectivity of the arrays and the reflectivity of the silicon substrate for which the absolute reflectivity is well characterized.

SHG measurements were performed in reflectivity with a $45^{\circ}$ incidence angle. The light source was a Ti:sapphire Coherent Mira femtosecond laser delivering pulses at the frequency of $85 \mathrm{MHz}$ for spectral range between 780 and 850 $\mathrm{nm}$. The time width of the pulse was $100 \mathrm{fs}$. The laser beam was slightly focused on the sample with a spot size of $100 \mu \mathrm{m}$. A set of red RG 715 filters was located just before the sample to drastically reduce the SHG from the optics. A set of three blue BG 40 filters was positioned just after the sample to reduce the fundamental beam (around $800 \mathrm{~nm}$ ) by a factor of $3.7 \times 10^{16}$ and transmit the $\mathrm{SH}$ beam (around 400 $\mathrm{nm}$ ) with a $61 \%$ efficiency. In both the linear and nonlinear experimental setups, the polarization was fixed transverse electric ( $S$-polarization). Two orientations of the sample were studied: incidence plane parallel or perpendicular to the chain of nanoparticles to get the electric field parallel (the $x$-direction) or perpendicular to the chains (the $y$-direction).
The surface symmetry of silicon was measured by rotating the sample to ensure a good alignment and that only SHG was measured. ${ }^{16}$

\section{RESULTS AND DISCUSSION}

Figure 1(b) shows the spectra in linear reflectivity. For each orientation of the electric field, a maximum is observed but at different spectral positions (at 790 and $930 \mathrm{~nm}$ for $X$ and $Y$-polarizations, respectively). The study on this shift on linear reflectivity is not the purpose of this paper, but we just report that due to the large spacing between the nanoparticles in the $Y$-direction and their interconnection along the $X$-direction; a stronger coupling between the resonant nanoparticles was expected for the electric field along the $X$-direction. This is in agreement with studies that demonstrated that interconnected nanoparticles behave differently than closely spaced nanoparticles. When the distance between the nanoparticles decreases (but the nanoparticles are still kept spaced), only a redshift of the dipolar resonance is observed. 3,4 As soon as they become connected, there is a "blueshiftlike" behavior due to the quadrupolar resonance at shorter wavelengths. ${ }^{4}$ Moreover, near-field microscopy investigations reported that the local electric field is enhanced within the gap between spaced nanoparticles when the gap is small enough to allow a strong near-field coupling. ${ }^{17}$ In the case of interconnected nanoparticles, a similar enhancement in the electric field at the nanobridge is expected and supported by our rigorous coupled wave method (RCWM) calculations.

Figure 2 presents the SHG signal at $400 \mathrm{~nm}$ (fundamental beam at $800 \mathrm{~nm}$ ) from the array for the two different orientations (with a $45^{\circ}$ incidence angle and TE polarization). Figures 2(a) and 2(b) represent an $X Y$-scan with a $50 \mu \mathrm{m}$ step on which we can resolve spatially the SHG signal of the two arrays (without and with defects). Initially, we will comment the results on the array without defects. After subtracting the SHG background from the substrate [about 100 counts from Figs. 2(a) and 2(b)], a SHG enhancement of 34 times is measured when the incident beam is polarized in the $X$-direction compared to the $Y$-direction (2924 and 86 counts, respectively, after subtracting the background). This is directly due to the spectral position of the maxima presented in Fig. 1(b). For comparison, in linear reflectivity, the signal was a factor of 0.85 lower for the $Y$-polarization compared to the $X$-polarization. This means that the enhancement in the SHG signal does not only come from higher reflectivity due to the spectral position but also from the enhancement in the localized electric field at the surface of the nanoparticles at the resonance.

Figure 3 shows the RCWM calculations of the electric field magnitude at the surface (gold-air interface on top of the nanoparticles) for these two orientations at $800 \mathrm{~nm}$. Numerical simulations were performed using MATLAB $® 6$ code taking into account 35 harmonics. The nanoparticle shape was approximated by a rectangle with the same dimension as measured from the SEM images $(50 \mathrm{~nm}$ thick, 145 $\times 160 \mathrm{~nm}^{2}$ ). The nanobridge is $15 \mathrm{~nm}$ long and $60 \mathrm{~nm}$ large. The distance between the chains in the $Y$-direction is 200 

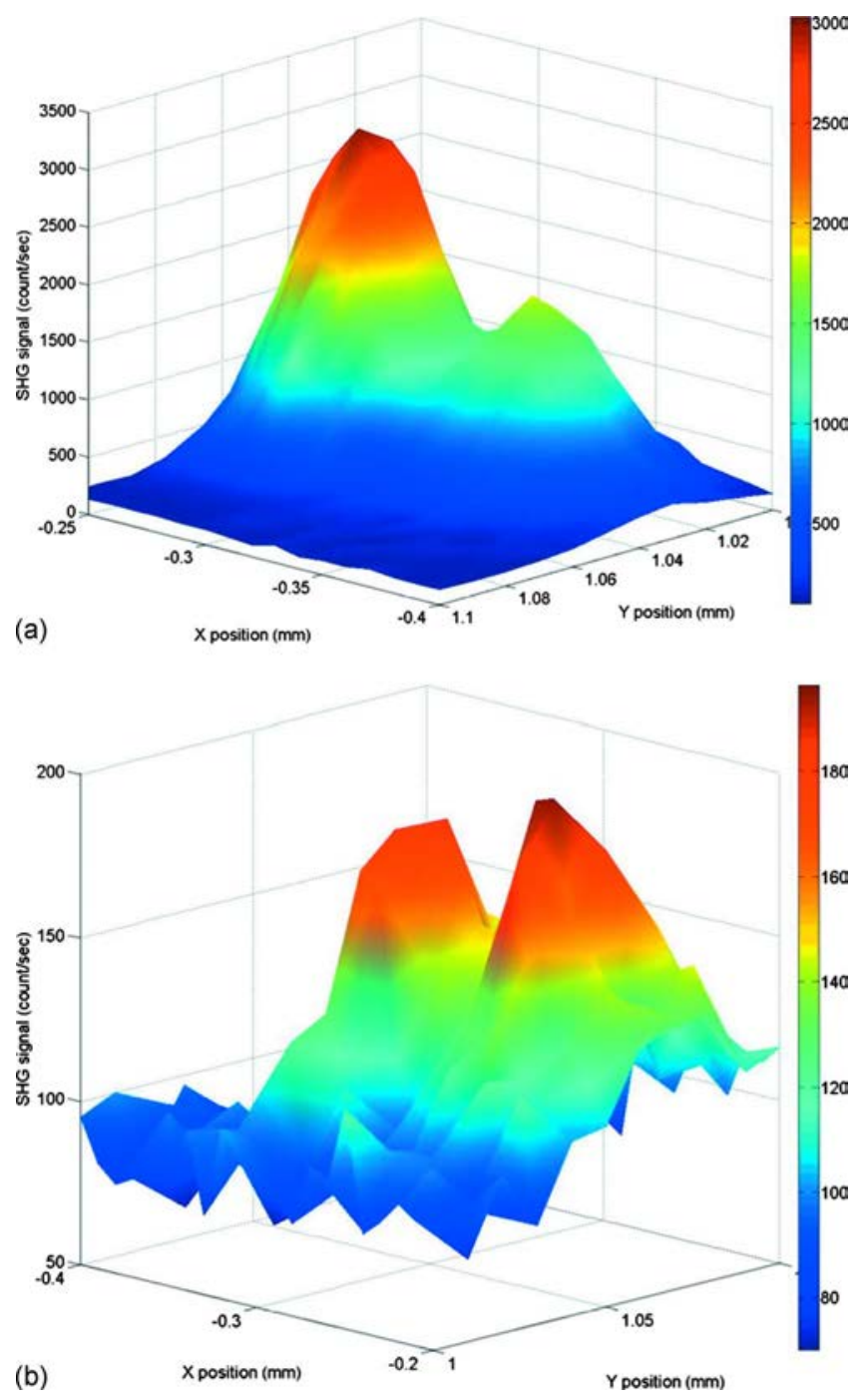

FIG. 2. (Color online) SHG signal from the arrays of gold nanoparticles. (a) With the electric field along the chains of nanoparticles and (b) with the electric field perpendicular to the chains of nanoparticles.

$\mathrm{nm}$. The electric field is strongly localized at the interconnection between the particles and is enhanced 20 times for $X$-polarization [Fig. 3(a)] compared to the $Y$-polarization [Fig. 3(b)], which should result in a SHG enhancement of 400 times. However, the SHG signal and the calculated electric fields should not be directly compared because SHG does not depend only on the electric field enhancement but also on the broken inverted symmetry and light at two different wavelengths (fundamental and second harmonics). Furthermore, the field enhancement is localized at the nanoscale while our measurements average the entire array. Nevertheless, these calculations confirm that the electrical connectivity supported by the gold nanobridge induces a strong coupling between adjacent nanoparticles. The RCWM calculations and their comparison with the SHG measurements suggest qualitatively that the strong localization of the electric field at the nanobridge plays an important role in the $\mathrm{SH}$ signal enhancement. This demonstrates that SHG is directly dependent on the localized electric field intensity rather than on the linear reflected light intensity.
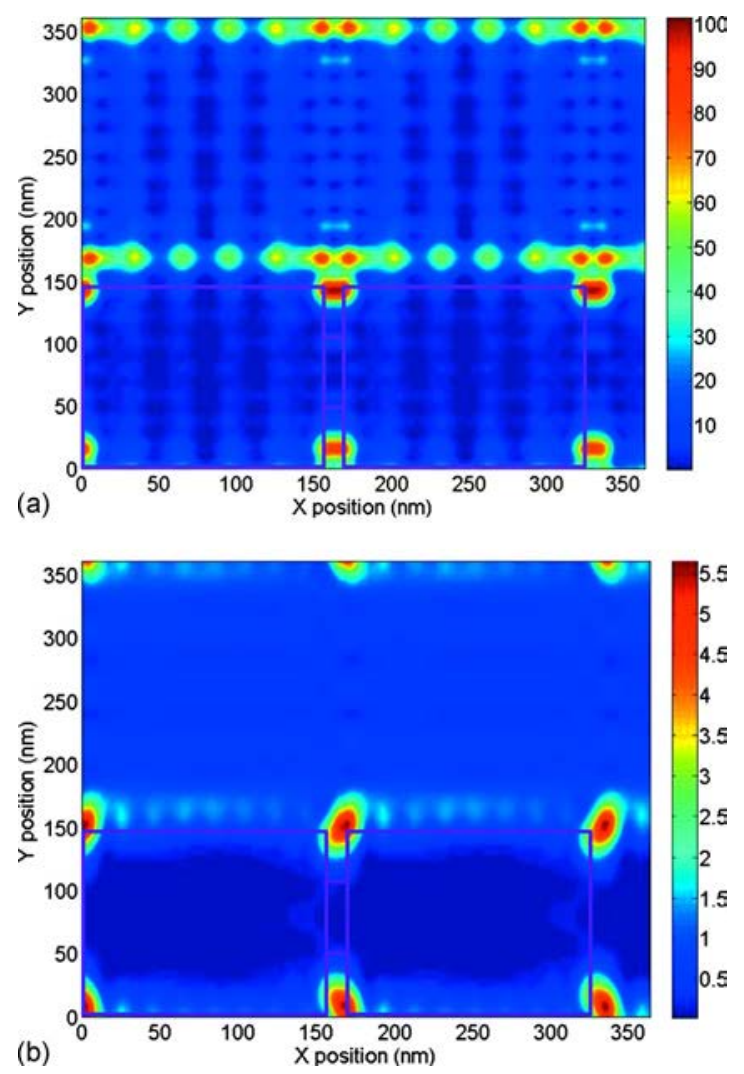

FIG. 3. (Color online) RCWM calculations showing the electric field map for one period with (a) the electric field along the chains of nanoparticles and (b) the electric field perpendicular to the chains of nanoparticles.

To study the range of the coupling evidenced by SHG, a second array with $10 \%$ random defects (missing nanoparticles) was also investigated. As shown in Figs. 2(a) and 2(b), it is remarkable to note that the signal in the two arrays (with or without defect) is at the same level for the $Y$-polarization, whereas there is a strong decrease for the $X$-polarization. This last configuration is interesting because we did not observe the same variation between the two arrays in the linear signal as well. Figure 4 shows the spectral variation in the ratio between the optical signal from the arrays with and without defects for both the SHG and the linear measurements with the polarization of the electric field along the chains. While the linear signal varies slightly around a factor of $100 \%$, there is an enhancement in this ratio for the SHG signal. This SHG ratio reaches a maximum value of $190 \%$ for the fundamental beam at $830 \mathrm{~nm}$ to be compared with the sample with defects. Also, this ratio depends on the fundamental wavelength, suggesting that it is related to a specific resonance of the chain. Indeed, the ratio is equal to $120 \%$ at $790 \mathrm{~nm}$, increases up to $190 \%$ at $830 \mathrm{~nm}$, and decreases to $140 \%$ at $855 \mathrm{~nm}$. While the ratio of $120 \%$ at $790 \mathrm{~nm}$ corresponds to the square of the lack of electric field supported by the $10 \%$ missing nanoparticles, the ratio of $190 \%$ at $830 \mathrm{~nm}$ hints at a more collective effect broken by the lack of nanoparticles in the chains. This enhancement shows that spectroscopic SHG reflectivity enhances an effect barely detectable by linear optical methods. The decrease in SHG with $10 \%$ defects also suggests that a rather long range propagating specific mode is strongly affected by these defects. Addi- 


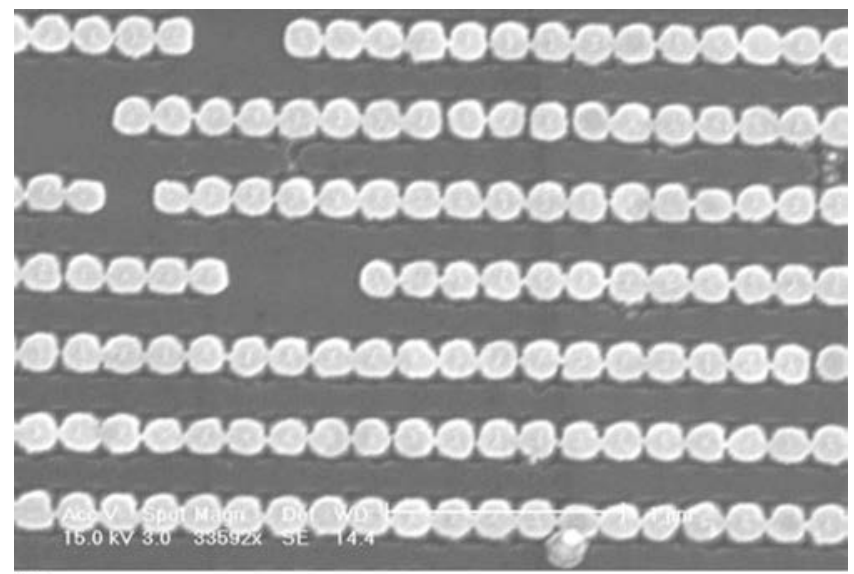

(a)

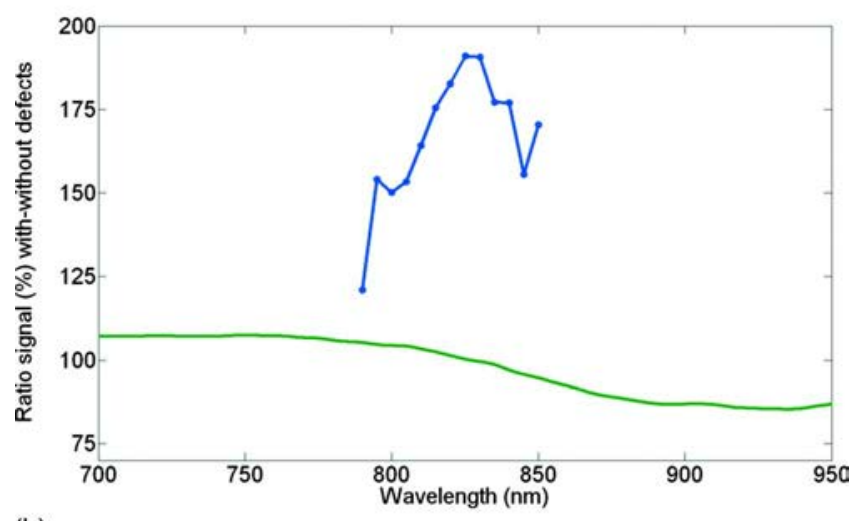

(b)

FIG. 4. (Color online) (a) SEM image of the array with $10 \%$ random defects. (b) Ratio of the SHG and linear optical reflectivity between the array without and with defects.

tional experimental investigations with different lengths of coupled nanoparticles are beyond the scope of this paper and will be addressed in the future.

\section{CONCLUSION}

In summary, we have demonstrated that SHG is a powerful tool to investigate the localization of SP fields at the nanoscale. SHG is not only proportional to the linear signal, but it is also directly influenced by the intensity of the local electric field at the interfaces. Furthermore, while linear spectroscopy cannot really bring significant information about the influence of defects in the array and on the local electric field intensity, in some cases, SHG can bring complementary information. This latter point could be further investigated in the light of the recent theoretical work provided by Markel and Sarychev ${ }^{18}$ about the impact of disorder on the SP properties within a linear chain of nanoparticles.

${ }^{1}$ S. A. Maier, M. L. Brongersma, P. G. Kik, and H. A. Atwater, Phys. Rev. B 65, 193408 (2002).

${ }^{2}$ N. Felidj, J. Aubard, G. Levi, J. R. Krenn, G. Schider, A. Leitner, and F. R. Aussenegg, Phys. Rev. B 66, 245407 (2002).

${ }^{3}$ L. Gunnarsson, T. Rindzevicius, J. Prikulis, B. Kasemo, M. Käll, S. Zou, and G. C. Schatz, J. Phys. Chem. B 109, 1079 (2005).

${ }^{4}$ T. Atay, J.-H. Song, and A. V. Nurmikko, Nano Lett. 4, 1627 (2004).

${ }^{5}$ J. R. Krenn, J. C. Weeber, A. Dereux, E. Bourillot, J. P. Goudonnet, B. Schider, A. Leitner, F. R. Aussenegg, and C. Girard, Phys. Rev. B 60, 5029 (1999).

${ }^{6}$ M. Salerno, J. R. Krenn, A. Hohenau, H. Ditlbacher, G. Schider, A. Leitner, and F. R. Aussenegg, Opt. Commun. 248, 543 (2005).

${ }^{7}$ S. A. Maier, M. L. Brongersma, P. G. Kik, S. Meltzer, A. A. G. Requicha, and H. A. Atwater, Adv. Mater. (Weinheim, Ger.) 13, 1501 (2001).

${ }^{8}$ U. Kreibig and M. Vollmer, Optical Properties of Metal Clusters (Springer, Heidelberg, 1995).

${ }^{9}$ G. Tessier and P. Beauvillain, Appl. Surf. Sci. 164, 175 (2000).

${ }^{10}$ K. H. Bennemann, Nonlinear Optics in Metals (Clarendon, Oxford, 1998).

${ }^{11}$ S. I. Bozhevolnyi, J. Beerman, and V. Coello, Phys. Rev. Lett. 90, 197403 (2003).

${ }^{12}$ X. M. Hua and J. I. Gerstan, Phys. Rev. B 33, 3756 (1986).

${ }^{13}$ C. Hubert, L. Billot, P.-M. Adam, R. Bachelot, P. Royer, J. Grand, D. Gindre, K. D. Dorkenoo, and A. Fort, Appl. Phys. Lett. 90, 181105 (2007).

${ }^{14}$ M. Airola, Y. Liu, and S. Blair, J. Opt. A, Pure Appl. Opt. 7, S118 (2005).

${ }^{15}$ A. Lesuffleur, L. K. S. Kumar, and R. Gordon, Appl. Phys. Lett. 88, 261104 (2006).

${ }^{16}$ W. Cheikh-Rouhou, L. C. Sampaio, B. Bartenlian, P. Beauvillain, A. Brun, J. Ferre, P. Georges, J.-P. Jamet, V. Mathet, and A. Stupakewicz, J. Magn. Magn. Mater. 240, 532 (2002).

${ }^{17}$ J. R. Krenn, A. Dereux, J. C. Weeber, E. Bourillot, Y. Lacroute, J. P. Goudonnet, G. Schider, W. Gotschy, A. Leitner, F. R. Aussenegg, and C. Girard, Phys. Rev. Lett. 82, 2590 (1999).

${ }^{18}$ V. A. Markel and A. K. Sarychev, Phys. Rev. B 75, 085426 (2007). 